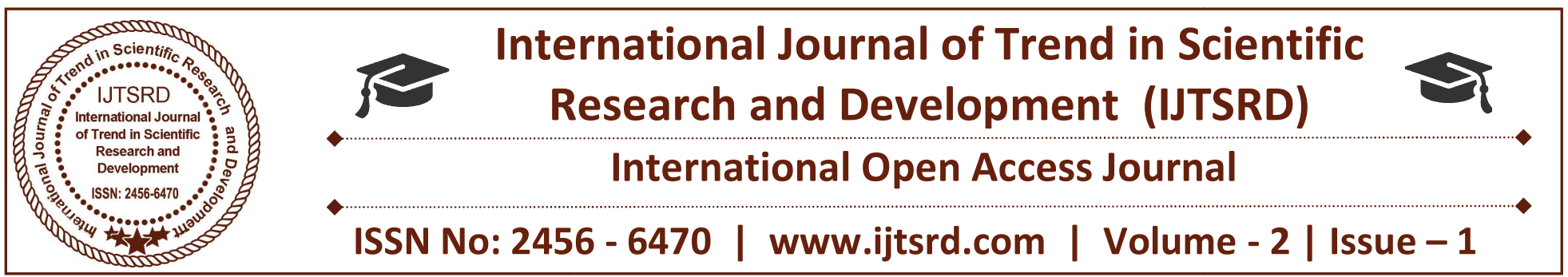

\title{
Video Retrieval Systems Methods, Techniques, Trends and Challenges
}

\author{
Mr. Rahul S Patel \\ ME E\&TC (Signal Processing), \\ JCOE Kuran Pune University
}

\author{
Mr. Gajanan P Khapre \\ ME E\&TC (Signal Processing), \\ JCOE Kuran Pune University
}

\author{
Prof. Mr. R. M. Mulajkr \\ PG Coordinator ME E\&TC \\ (Signal Processing), Dept JCOE \\ Kuran Pune University
}

\section{ABSTRACT}

Content primarily based Video Retrieval (CBVR) has been increasingly accustomed describe the method of retrieving desired videos from an oversized assortment on the premise of options that are extracted from the videos. The extracted options are accustomed index, classify and retrieve desired and relevant videos whereas filtering out unwanted ones. Videos are often pictured by their audio, texts, faces and objects in their frames. An individual video possesses distinctive motion options, color histograms, motion histograms, text options, audio options, features extracted from faces and objects existing in its frames. Videos containing helpful info and occupying significant house within the databases are under-utilized unless CBVR systems capable of retrieving desired videos by sharply choosing relevant whereas filtering out unwanted videos exist. Results have shown performance improvement (higher precision and recall values) once options appropriate to particular kinds of videos are used with wisdom. Various combinations of those options also can be accustomed reach desired performance. During this paper a fancy and wide space of CBVR and CBVR systems has been bestowed in a very comprehensive and easy approach. Processes at completely different stages in CBVR systems are represented in a very systematic approach. Types of options, their mixtures and their utilization ways, techniques and algorithms are shown. Numerous querying methods, a number of the options like GLCM, Dennis Gabor Magnitude, and algorithm to get similarity like Kullback-Leibler distance method and relevancy Feedback technique are mentioned.
Keywords: VR, GLCM, Gabor Magnitude, KullbackLeibler Distance Method, Relevance Feedback Method

\section{INTRODUCTION}

In these days"s digital global massive amount of useful digital facts like pictures, audio and video records apart from textual information exists on-line and is to be had to public, government authorities, experts and researchers very effortlessly and on hand at fairly inexpensive fee because of fast increase in availability of person pleasant and inexpensive Multimedia acquisition gadgets at a completely big scale like high decision camera in mobile telephones, available cams and different advanced virtual devices, availability of high capability garage devices like memory cards, difficult disks, and so forth., big scale usage of net by using hastily developing wide variety of applications utilized by digital gadgets to add big quantity of multimedia records, advanced web era an internet infrastructure [6], [7]. video facts possesses a number of information for those the usage of multimedia structures and programs like virtual libraries, guides, education, broadcasting and enjoyment. such programs are useful most effective while video retrieval systems are green enough to retrieve videos and other vital statistics from huge databases as quick as viable [2]. However, it's far extremely tough for the present internet engines like google to look for video over the web so novel methodologies are required that are able to manipulating the video facts according to the content material [13]. For multimedia mining, mixtures of 
multimedia statistics are saved and organized the usage of strategies like class and annotation of films [6], [15], [16]. maximum of the net based video retrieval systems paintings by means of indexing and looking films primarily based on texts associated with them but this method does not perform nicely due to the fact the texts do no longer comprise enough records of the videos [2]. seeing that video retrieval isn't always effective the use of conventional query-by way of-text retrieval approach, content material based totally video retrieval (cbvr) is considered as one of the great realistic solutions for higher retrieval great [6]. Because of exploitation of rich video content, there's a first-rate scope in place of video retrieval to enhance the performance of conventional search engines [7]. that is main the place of cbvr right into a direction promising to create greater powerful video seek engines in destiny [12].

In section 2 Processes and components of CBVR systems are elaborated; section 3 shows the methodology to obtain results in CBVR systems. Different types of CBVR systems are given in section 4 , problems and challenges posed to information retrieval and CBVR systems are discussed in section 5 and the conclusion is presented in section 6 .

\section{VIDEO RETRIEVAL SYSTEMS PROCESSES AND COMPONENTS}

\subsection{Formation of a Video}

A shot is a set of frames captured by using a digital camera constantly and a clip is the prevalence of such consecutive pictures. Consecutive pictures showing one of kind students strolling in unique schools of a university campus forms a clip of a campus [2].

\subsection{Segmentation of Video}

Step one in most of current content primarily based video evaluation techniques is to carry out segmentation of video into simple photographs. Those shots incorporate a series of frames recorded one after some other to shape a video occasion or scene constantly varying in time in addition to area. Those are organized and edited with cut transitions or gradual variant of visual consequences forming a video scene or sequence in the course of video sorting [7]. Therefore, process of video segmentation is not anything however changing a video into diverse smaller video clips representing different scenes where each scene is decomposed once more into one of a kind pictures containing large quantity of frames in every shot. Features are extracted from these components of video and are then exploited to save, classify, index and retrieve movies from big databases.

\subsection{Classification of Videos}

Classification of films enables to growth efficiency of video retrieval and its miles one of the maximum vital duties [1]. at some stage in system of video class [24], [25] information is received from functions extracted out of the video components, videos are then, positioned in categories defined earlier. facts which include visible and movement features of numerous additives of video like items, shots and scenes is obtained [1]. Maximum of the class techniques are both semantic content class and non-semantic content material category. The maximum appropriate one is employed as in line with the type of a video and alertness and accordingly, video can be labeled to the maximum suitable and closest among all predefined classes. Semantic video class can be completed at three levels of a video. video genres, video events and items within the video [26]. Video genres based classification is to categories videos into one of the pre-described categories of movies. These categories of films are types of videos typically exist like movies of sports, news, cartoons, films, flora and fauna, documentary movies, and many others. video genres based type has higher and broader detection capability at the same time as items and activities have narrow detection variety [26]. Event based video class is based totally on occasion detection in a video information and to categories it into considered one of the pre-described categories. an event is said to be passed off if it has vast and visible video content material. a video could have many occasions and every event has sub-occasions. one of the most critical steps in content material primarily based video category is to classify events of a video [17]. Photographs are maximum primary thing of a video [7]. Classification of pictures determines type of motion pictures. shots are classified using features of objects in pictures [19]. special forms of video features, motion, color, texture and aspect for each shot are extracted for video retrieval [7]. picture retrieval techniques and techniques may be used for key body based totally video retrieval systems [1]. Low level visible functions of key-frames are exploited for this cause [9]. in key-body based retrieval, as a video is abstracted and represented via 
capabilities of its key-frames, indexing techniques of picture database can be carried out to shot indexing. Every shot and all its key-frames are connected to each different. for a video retrieval, a shot is searched with the aid of identifying its key-frame [3], [4]. Computational cost

Worried even as the usage of all frames of a shot to retrieve a video is lots better than that after handiest key frames are used to represent a shot. visible functions of those key frames are compared with the ones of the videos in the database for retrieval [2]. Key-frames also are hired in face [11] and item primarily based video retrieval. a massive number of cbvr structures some of the current ones are working with keyframe. Key-frames can deliver quite a few beneficial facts for retrieval motive and if required, static functions of keyframe [20] can also be used to measure video similarity along with motion capabilities [22] and object functions [21]. Item based video type is primarily based on item detection in video information [18]. Faces and texts also are used as a way to categories films. four styles of television programs are labeled through approach proposed by dimitrova et al. [23]. Faces and texts are detected and then tracked to each frame of video segment. frames are categorized for a specific type in keeping with respective faces and texts. an hmm [14] (hidden markov version) is skilled to categories each kind of frame the usage of their labels. the arrival of textual information at the same time as streaming of video frames enables making an automated video retrieval gadget [10] primarily based on texts appearing in consecutive frames. Video class the use of gadgets such as faces and texts work simplest in precise environment and this class for video indexing has the obstacle that they are not common. object based video classification usually shows bad performance [1].

\subsection{Query of a Video}

Queries the usage of objects, sketches or example images do no longer make use of semantic information [1].

Query by using object: the item image is provided. The occurrences of objects in video database are detected and places of the object decide success of the question [18].

Query by way of text: as it's miles popular for content based totally photo retrieval, instance pics can be used as query to retrieve relevant motion pictures in a database of motion pictures (query by way of example) however it has a hassle that movement statistics of the video being searched isn't applied. it is predicated most effective on the appearance facts. Additionally, finding video clip for the interested idea may also grow to be too complex using instance photo. Textual question gives extra herbal interface and claims to be higher method for querying in video databases [10].

Query through instance: query by way of example is better if visible capabilities of the question are used for content material primarily based video retrieval [2]. Low stage capabilities are acquired from key frames [9] of the query video and then they may be in comparison to split out the same films the usage of their key frames visual features [1].

Query with the aid of shot: some structures utilize the entire video shot as the question as opposed to key frames [5]. this may be a higher alternative but with a better computational cost.

Query with the aid of clip: a clip may be used for better performance of video retrieval in comparison to the approach when a shot is used due to the fact a shot do now not represents sufficient data approximately the entire context. all of the clips which possess a enormous similarity or relevancy with the query clip are retrieved [2].

Query by means of faces and texts: faces and texts also can be used as a question to retrieve a video section containing frames categorized for a selected kind consistent with faces and texts [23]. A suitable algorithm can be used to look the video enquired by using the query clip the usage of information obtained from faces and texts in frames of the query clip.

\subsection{Features and Features Extraction}

For powerful video indexing, class and retrieval visual functions embedded in video records is exploited. Three primary functions to be extracted are shade, texture and motion for powerful video indexing. these functions are represented by coloration histogram, gabor texture functions and motion histogram respectively [5]. The most useful information in the videos includes functions of the objects, key frames and the motion capabilities [1]. key body functions: key frames in videos include coloration, texture and form based static features. Texture, shade and shape are most big visual properties and are primary concerns in low degree image and pc vision issues. 
various colour functions are color moments, shade histograms [75], colour correlograms[76] and the color capabilities obtained from a few gaussianmodels [1]. One-of-a-kind color capabilities are extracted for extraordinary styles of shade areas which include rgb, hsv, ycber and normalized r-g, yuv, and hvc. They play one of the most important roles for video indexing and retrieval. These features are extracted directly from an photograph or sometimes from sub blocks [77] of the partitioned photo. texture alone is a complicated studies problem. it represents an area by roughness, directionality, repeatability and variability features over a positive spatial extent at the same time as coloration is a point belongings in an photograph [7]. Texture functions are extracted by using locating strength distribution in frequency domain by way of specific strategies [39], [40], [41]. gabor wavelet features are acquired using one such technique to retrieve and classify photos and motion pictures [42]. Texture based totally features are functions representing specific occurrence pattern of items, homogeneity and agency of various items of diverse Shapes and their personal features, independent of depth and coloration, with varying heritage and their correlations with neighboring visible characteristics. exceptional texture functions are orientation capabilities, wavelet transformation primarily based texture features, tamura functions, co-incidence matrices, simultaneous autoregressive fashions, etc., [1]. Tamura functions are six texture based capabilities corresponding to human visual perception: coarseness, contrast, directionality, linelikeness, regularity, and roughness. The primary 3 features are vast for human belief and are accountable to differentiate distinct textures [80]. A co occurrence

Matrix is a matrix or distribution of co-going on values for a picture [81]. It represents texture in photographs. The matrix elements are the counts of the variety of instances a given feature occurs in a particular spatial relation to any other given feature [82]. A co-occurrence matrix can use any of the Functions from the photo. Glcm is the co-occurrence matrix whilst grey degree is selected as a characteristic. the glcm is a tabulation of how often specific mixtures of pixel gray levels occur in an photo. an instance to discover glcm of a matrix of fig. 1 having gray values zero, $1,2,3$ are proven here

\begin{tabular}{|l|l|l|l|}
\hline 0 & 0 & 1 & 1 \\
\hline 0 & 0 & 1 & 1 \\
\hline 0 & 2 & 2 & 2 \\
\hline 2 & 2 & 3 & 3 \\
\hline
\end{tabular}

Fig 1: Matrix
And its GLCM is shown in fig. 2

\begin{tabular}{|l|l|l|l|l|}
\hline $\begin{array}{l}\text { Nelghbour } \\
\text { pixel } \\
\text { values }\end{array}$ & 0 & 1 & 2 & 3 \\
$\begin{array}{l}\text { Reference } \\
\text { pixel values }\end{array}$ & 2 & 2 & 1 & 0 \\
\hline 0 & 0 & 2 & 0 & 0 \\
\hline 1 & 0 & 0 & 3 & 1 \\
\hline 2 & 0 & 0 & 0 & 1 \\
\hline 3 & & & & \\
\hline
\end{tabular}

Fig 2: GLCM of the matrix of fig. 1

Texture capabilities can be applied correctly for video retrieval purpose [1]. Hauptmann et al. [38] use gabor wavelet filters to acquire texture features for video search engine. They layout 12 oriented electricity filters. a texture feature vector is fashioned with the imply and variance of the filtered outputs.

the photo is split into small blocks and gabor clear out is used to reap capabilities from these blocks [47]. Hauptmann et al. [46] divide the photo into blocks each of size $5 \mathrm{x}$ five and compute texture functions from every block the use of gaborwavelet

filters. gabor texture features have proven better performance than different texture features [43]. object shapes and their functions are received from edges and nearby capabilities of numerous items the usage of histogram [1]. an edge histogram descriptor (ehd) is designed [78], [79] by using dividing an image into $4 \times 4$ blocks (sixteen sub-pix). The spatial distribution of edges is acquired after which, categorized into five unique orientations of zero, 45, ninety, a hundred thirty five levels and a ,nondirectional" facet in each block. the end is the variety of pixels forming an edge of a specific class. the output end is a 5 bin histogram for each block, getting a complete of eighty (5x16) histogram bins. Movement capabilities: the characteristic of dynamic films that distinguishes them from nevertheless photographs is the movement of objects and motion of historical past towards each different. the foreground motion is because of shifting objects whereas the background motion is because of dig cam movement. Visual content with temporal variation is represented by using movement functions. Monitoring of transferring object (motion detection) is essential in video retrieval systems. it includes isolating and finding which pixels belong to moving objects and the pixels belonging to static heritage over a length of 
time [83]. the difference between a video and an image is the motion as movement functions deliver semantic principles as In comparison to object and key body features in an photo [1]. video motion is of two kinds, historical past movement and foreground motion due to dig cam motion and object' $s$ movement respectively. Therefore, types of movement functions are to be had. dig cam primarily based movement features encompass features as a result of zooming in or out, panning left or proper and tilting up or down by means of digital camera. object primarily based movement features are more critical as they may be able to describe motions of key gadgets. Motion features are used to classify shots and are hired for shot boundary detection using cuts, sluggish and no trade frames [84], [85], [86]. Motion features also are hired to acquire key frames by dividing a shot into segments with identical cumulative motion pastime The use of mpeg-7 motion interest descriptor. Key body is the frame placed inside the middle of each segment [87]. a triangle model of movement strength for movement patterns in movies was proposed [88] wherein frames at the turning factors of the movement acceleration and motion deceleration are selected as key frames. Movement is the critical visual characteristic carrying temporal variation of video. The correlation between body sequences inside a video shot is a few of the motion functions. Movement information of a video is obtained by dimensional motion histogram of the movement vectors and the colour histogram [2]. The displacement in horizontal and vertical guidelines are quantized into 121 bins each (60 packing containers for high-quality, 60 for bad and one for zero). Totally, there are $121 \times 121$ packing containers for this 2-d motion histogram. Movement vectors are obtained between consecutive frames of mpeg-I video circulate. in mpeg video, each body is partitioned into blocks every of length $16 \times 16$ pixels referred to as macro blocks $(\mathrm{mb})$. Movement vector is defined because the displacement of the goal mb (modern frame) from the prediction $\mathrm{mb}$ (reference body). In mpeg layout there are $i, p$ and $b$ frames. $i$ frames aren't used for movement information. $p$ frames incorporate ahead movement prediction and $b$ frames comprise both ahead and backward movement prediction. Motion histogram is fashioned the use of motion vectors present in $\mathrm{p}$ frames. Their average price is acquired for removal of noise outcomes with the aid of normalizing them using wide variety of frames in a shot [2]. Object features: items are represented using capabilities of texture, shade and trajectory of the items [19]. Object features used for item based video retrieval are the colour, length, texture features of the areas inside the objects [1]. They can be used to retrieve motion pictures in all likelihood to contain similar gadgets [34]. Faces are also used to retrieve motion pictures as objects in lots of video retrieval structures. as an example, sivic et al. [35] construct retrieval gadget of someone that is able to retrieve shots containing that individual, given a question face in a shot. Photographs are ranked as according to the similarity measure. le et al.

[36] Endorse a method to retrieve faces in broadcast news movies by way of integrating temporal data into facial depth data. Texts can also be used as gadgets and make a contribution in conjunction with faces for video retrieval. li and doorman [37] put in force textbased video indexing and retrieval by increasing the semantics of a query and using the glimpse matching approach o carry out approximate matching instead of exact matching. Problem of object based features is that plenty of time is consumed for searching and identifying the gadgets within the motion pictures [1]. Broadly various types of features are employed by huge variety of strategies to constitute [7], classify, enquire and retrieve motion pictures. Among Those, most popularly used capabilities [7] are textual content evaluation [30], form information [28], colour histogram [27] and movement hobby [29]. a aggregate of various types of capabilities i.e., object features [21], static functions of key frames [32], and motion capabilities [22] can be used to discover similar video while demanded through user [1]. Edge histogram and texture functions are one of the most reliable information for powerful video retrieval utility. Textural houses of texts are wonderful and distinguish them from its background inside the photograph. This can be exploited by way of texture primarily based strategies to retrieve texts from photographs. Texture functions of the location in an image containing texts may be obtained by way of techniques using Fourier transform, spatial variance, wavelet remodel and gabor Filters [10]. Extraction of Gabor capabilities: Gabor filters are a collection of wavelets, with each wavelet shooting power at a particular frequency and a specific course. Expanding a signal the use of this foundation offers a localized frequency description, consequently capturing local features/strength of the sign. Texture functions can then be extracted from this organization of strength distributions. the dimensions (frequency) and orientation tunable belongings of gabor filter makes it especially useful for texture analysis. The filters of a gabor clear out 
bank are designed to hit upon one-of-a-kind frequencies and orientations. They may be used to extract capabilities on key points detected via hobby operators [72]. from every filtered picture, gabor functions can be calculated and used to retrieve pix. The algorithm for extracting the Gabor characteristic vector is proven in fig. three and the related equations $(1-4)$ are also shown beneath

[73], [89]. For a given photograph i(x,y), the discrete gabor wavelet transform is given via a convolution:

$$
W_{m n} \sum_{x 1} \sum_{y 1} I\left(x_{1} y_{1}\right) g_{m n} *\left(x-x_{1}, y-y_{1}\right)
$$

Where $\square$ indicates complex conjugate and m, n specify the scale and orientations of wavelet respectively. After applying Gabor filters on the image with different orientation a different scale, an array of magnitudes is obtained:

$$
E(m, n) \sum_{x} \sum_{y}\left|W_{m n}(x, y)\right|
$$

These magnitudes represent the energy content at different scale and orientation of the image. The main purpose of texture-based retrieval is to find images or regions with similar texture.

The standard deviation $s$ of the magnitude of the transformed coefficients is:

$$
\sigma_{m n} \sqrt{\frac{\sum_{x} \sum_{y}\left(\left|W_{m n}(x, y)\right|-m n\right)^{2}}{P X Q}}
$$

Where $\mu$ is the mean of magnitude and given as $m n$ $\frac{E_{m, n}}{P Q X}$

A feature vector $\mathrm{f}$ (texture representation) is created using $\mathrm{mn}$ as the feature components [74], [68]. M scales and $\mathrm{N}$ orientations are used and the feature vector is given equation (4)

$$
f\left[\sigma_{00}, \sigma_{01}, \sigma_{02} \ldots . \sigma_{(M-1)(N-1)}\right]
$$

$f_{\text {Gabor }} \frac{f^{-}}{\sigma}$ Where $\mu$ is the mean and $\sigma$ is standard deviation of $f$.

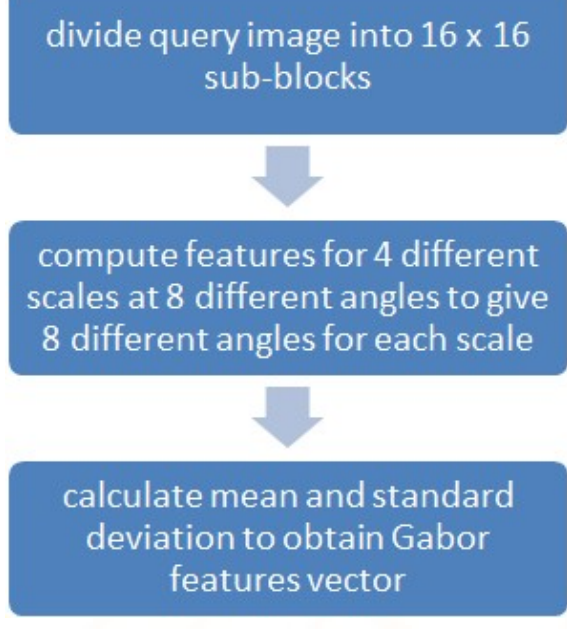

Fig 3: Gabor Filter Algorithm

\subsection{Similarity Measure}

Queries are categorized through classes taken care of out in step with form of capabilities used or form of example data. the question is determined out through calculating similarity between feature vector [44], [45] stored inside the database and the query functions. The similarity is received with the enquired nevertheless image, still pix from example video clip, gadgets, texts or a particular face from still pictures or video clip, motion capabilities from example video [11]. picture similarity matching for example based totally picture retrieval has been studied for many years. The picture seek engine finds an picture from a database with the help of similarity between characteristic vectors via a distance between them. Commonly Euclidean distance is measured to locate similarity. Similar pictures are ranked as according to the space among the query image and snap shots from database. kullback-leibler distance approach is also employed for the similarity measure between question features and the features from the feature library [7]. Sorts of functions decide the overall performance of video retrieval gadget. once features are generated overall performance may be greater with higher consequences from similarity degree by knowing more accurately about figuring out how plenty close or a long way is the retrieved result. Euclidean distance and Murkowski type distances are significantly used [7]. Video retrieval result depends substantially on video similarity measures. The films are retrieved by measuring similarity between the question video and motion pictures from the database. the similarity can be acquired via matching their functions, texts, objects, faces, etc. and their combinations. Measuring similarity by using 
matching capabilities is maximum convenient and direct method [1]. It's far measured through the average distance between features of corresponding frames [48]. in question with the aid of instance similarity measure to locate relevant videos commonly low degree function matching is used. Video similarity may be measured at one of kind levels of resolution or granularity [49]. a video clip is retrieved via locating key frames happening sequentially within the video database that are Just like that of the question video [2]. a query frame can also receive to a gadget to retrieve similar movies from the database. The distance metric is called as similarity measure whereas in traditional retrieval machine, the Euclidean distance among the question and database is calculated to rank the retrieved videos. the video from the database similar to the body just like the question frame is higher in rank if the Euclidean distance is smaller [4], [10]. The equation for Euclidean distance between the query photo $q$ and an photo $\mathrm{p}$ is proven in equation(5)

$$
E D \sum_{i 1}^{n} \sqrt{V_{p i}-V_{q i} . V_{p i}-V_{q i}}
$$

Where $V_{\mathrm{pi}}$ and $\mathrm{V}_{\mathrm{qi}}$ are the feature vectors of Query image $\mathrm{Q}$ and image $\mathrm{P}$ respectively of size , $\mathrm{n}^{\mathrm{ee}}$. Apart fromEuclidean Distance, there are many other methods to measure feature distance between two images like Manhattadistance, the Mahalanobis Distance, Earth Mover s Distanc(EMD) and the chord distance [33]. Kullback and Leibler determined similarity measure based on two probabilitdistributions associated with the same experiment [31] i.e. same event space. KullbackLeibler divergence measure is used to find the difference between two distinct probability distributions [7]. The equation for KL divergence of the probability distributions $\mathrm{F}, \mathrm{G}$ on a finite set $\mathrm{P}$ is given in equation (6).

$$
D_{K L} F / / G \sum_{p \in P} F(p) \log F(p) / G(p)
$$

Below are the steps for Similarity Measure: Let us consider -F as Query clip feature vector, $\mathrm{G}$ as Feature library $1^{\text {st }}$ feature vector, $i$ as Element of vector, $M$ as Normalized factor of $\mathrm{G}$

$$
V \frac{F}{\operatorname{Normalization}(F)}
$$

Then find ((G 0) \& ( $\mathrm{V} 0)$ ) and store that in $\mathrm{V}$ Then similarity measure is carried out using equation (8)

$$
D_{K L} \sum V V_{A} \log \frac{M * V V_{A}}{G V_{A}}
$$

Neural Network can also be used to find similar shots. It is used to cluster shots and hence classify videos to the best matching cluster based on features obtained from its shots. The features of color, texture and trajectory of objects in a shot are used to map the shot to the best matching cluster [19] in object-based query. Similarity between the query image I and an image $I$ in the video database is obtained by probability of generating the image I given the observation of the query image I G [1].

\section{RESULT EVALUATION}

The overall performance of video retrieval is evaluated with the same parameters as it's far evaluated in photograph retrieval [47]. Consider and precision are the 2 parameters [2] as given in equations (9) and (10).

$$
\begin{aligned}
& \text { Recall } \frac{D C}{D B} \\
& \text { Precision } \frac{D C}{D T}
\end{aligned}
$$

$D C=$ number of similar clips detected correctly

$D B=$ number of similar clips in $t \square$ e detabase

$D T=$ total number of detected clips

\section{VIDEO RETRIEVAL SYSTEMS}

Video retrieval techniques are widely distributed among two types. One in every of them is comparison of frames and their corresponding functions inside clips. A set of frames is received which can be sequentially matching which allows inside the retrieval of motion pictures. This approach is easy however the computational cost depends upon the functions length and may be very excessive Further with that, these techniques have a drawback of synchronization between frames as exceptional clips may additionally have used one-of-a-kind charge to encode them. To triumph over the disadvantage of the above strategies a key body is used to represent an entire shot. Shot matching is executed and as a result video retrieval is finished by means of comparing their features. Drawback of strategies using key 
frames matching is that temporal data and the associated data among the important thing frames in a shot are misplaced. Finding a suitable key body is difficult to select. To strike a balance among the performance and computational cost, more visual capabilities are used from the frames to symbolize a shot [2]. It's far learnt from the evaluation of video information retrieval that properly image retrieval ends in suitable overall performance of video retrieval system whilst question is an photo or an image from the query video [11]. A huge wide variety of tactics were experimented for indexing, type and retrieval of movies from large video databases. The video content is represented by spatial and temporal characteristics of movies. In spatial area, capabilities are received from frames to shape characteristic vectors from specific elements of the frames. In temporal domain, video is segmented into its elements like frames, shots, scenes and video clips and features like histograms, moments, textures and motion vectors represent the data content of these Video segments [10]. An average technique is utilized in gadget proposed wherein a video is retrieved based on a question clip [7]. Right here, database is processed offline. They used 2-D correlation coefficient approach together with discrete cosine transform, imply and well-known deviation over video sequences for segmentation of videos from database into primary shots. Every video shot is represented by means of four types of capabilities. Colour, texture, aspect and movement feature which is the characteristic representing temporal statistics of movies. These functions from the query clip are in comparison with capabilities inside the database. Kullback-Leibler method is used to degree similarity. Video sequences are ranked consistent with the distance measures and similar films are retrieved. As stated above, clip based totally retrieval yields higher effects than that when simplest key frames representing a shot is used. So, it is higher to apply complete video shot instead of key frames

as the question [5]. Broadcast information video database has sizeable data. The presence of textual captions with audio and video records makes this system an effective textual based automatic retrieval machine which gives important statistics get right of entry to thru retrieving news movies [10]. Face detection is classified for picture and video evaluation. It changed into experimented in a commercial machine [70]. It was found that accuracy of face reputation in video series of the sort referred to in the machine [11] become too poor to show to be beneficial. normal a large variety of queries do no longer yield excellent effects as cited [11] about one third of the queries had been unanswerable with the aid of any of the automated systems taking part in the video retrieval music[71]. No machine or method became capable of provide applicable outcomes. An incorporated video retrieval gadget is proposed [2] in which a video shot is represented now not by means of key frame only but via all frames to extract A process waft of a typical CBVR machine is shown in fig. 4. A video thing i.e., frames, pictures or scenes, and so on. Are extracted from motion pictures after which categorized to pre-described classes. Class to these categories is performed manually. Capabilities are then extracted for each component and stored in features database. Functions of the identical component from the question video also are extracted and then in comparison with capabilities saved inside the database. The output video is acquired by using locating the similarity measure between functions of query video and the functions saved within the database.

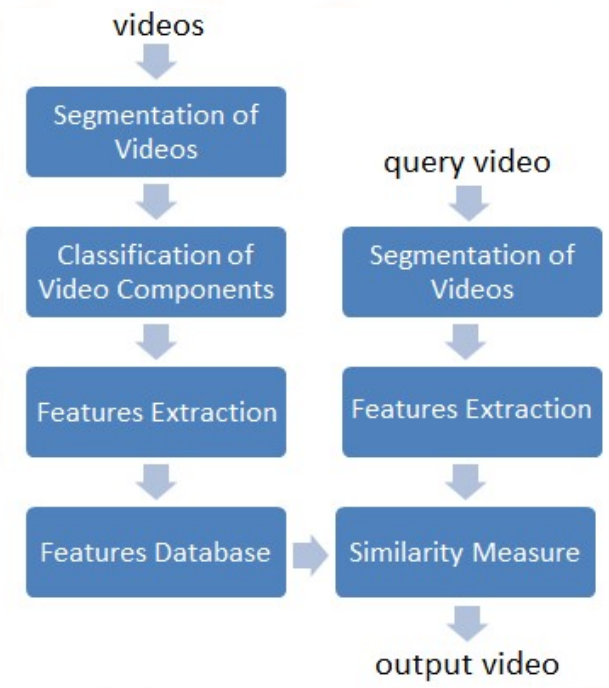

Fig.4: VR System

Fig 4:VR system to improve the retrieval overall performance, relevance feedback technique can be used to resemble human visible judgment and similarity belief up to a certain volume. Systems using relevance remarks are effective in rating and retrieving similar motion pictures. It eliminates the distinction between low degree features and semantic concept of the films [1]. It relies on comments acquired by way of user or can be automatic and accordingly the videos are ranked. The ranking and the feedback is used to enhance similarly searches. A relevance feedback system retrieves initial consequences through using conventional strategies like question by means of example picture, etc.then, 
the user will offer feedback to the device regarding relevancy of the retrieved end result with the query. The feedback will assist to improve the retrieval satisfactory. it's miles a compromise between a completely automatic, unsupervised system and system based on user's feedback due to the fact a system learning algorithm may be used to examine the user's comments [8]. because it is not smooth to fill the distance between low level capabilities and highlevel principles for each sort of query, video retrieval based on this mapping is difficult. Additionally, greater human involvement yield one of kind effects under different instances. To tackle those troubles a relevance remarks which adjusts its weight according to user's remarks iteratively to fill the gapso that excessive level principles may be represented by means of low level features. Relevance comments are used within the device [2]. The result is acquired with the aid of updating the values of $\mathrm{Mu}$ and updating of $\mathrm{Mu}$ is finished by using approach proven beneath.

$$
\begin{gathered}
M_{u}\left\{M_{u} \text { Score }_{v} \text { if }_{v}^{u} \in S\right. \\
M_{u}\left\{M_{u} 0\right. \text { otherwise } \\
v 1,2, \ldots \ldots . L \\
u x, y
\end{gathered}
$$

Weights $M x$ and $M y$ are updated using user's feedback. Let $\mathrm{S}$ be the set containing the most similar $\mathrm{L}$ retrieved video clips, overall similarity value $H y$ And value of $M x$ and $M y$ is 0.5 .

$$
\begin{gathered}
S S 1, S 2, \ldots \ldots . S L \\
\text { Score } \quad \text { Score } 1, \text { Score } 2, \ldots \ldots \ldots \ldots \text {....... }
\end{gathered}
$$

be the set containing scores by relevance feedback by the user for each retrieved clips in set $\mathrm{S}$. The scores may have any of the values from $-3,-1,0,+1$, and 3 . Where these values correspond to the feedback as

$$
\begin{aligned}
+3 & \rightarrow \text { highly relevant } \\
+1 & \rightarrow \text { relevant } \\
0 & \rightarrow \text { no opinion } \\
-1 & \rightarrow \text { non-relevant } \\
-3 & \rightarrow \text { highly non-relevant. }
\end{aligned}
$$

\section{PROBLEMS AND CHALLENGES}

With loss of delight from textual primarily based video retrieval, the concept of content material primarily based video retrieval has been the interest for researchers because long time. in the beginning of content based totally video retrieval, they attempted to retrieve movies the usage of an picture. However, video retrieval using query with the aid of image is not a hit as it can't constitute a video. A video is a sequence of pictures and audio. A query video gives wealthy content material facts than that supplied by way of a question picture. Locating the applicable video with the aid of sequentially comparing the low level visual features of key frames of the query video with the ones of key frames of films in database offer lengthy pending option to yield higher end result[9] of video retrieval. Finding similarity degree requires key frames matching and hence computing key body features including coloration histogram, texture and side functions, and so forth. To calculate distance parameter. These large computations reason lengthy response time to the customers and hence, the hassle of excessive computation fee in computing visible functions of movies is persistent. Aside from this, concerns for motion functions, temporal, series and period of shots in a video pose a undertaking for the studies area[6]. The structural and content material attributes obtained thru content material analysis, segmentation, video parsing, abstraction approaches and the attributes entered manually are called metadata. Video is listed on a table using the metadata the use of clustering manner that categorizes video clips or pictures. Clustering technique categorizes movies or pictures the use of metadata to form an index desk of movies into distinctive visual classes. Researchers have advanced numerous equipment and schemes to index, enquire, browse, search and retrieve movies from huge databases however effective and robust tools are still missing to test with massive databases [9]. due to these boundaries [6], [9] a majority of video searches and retrievals still is predicated on key-word or textual content attributions.

\section{CONCLUSION}

It is able to be concluded from discussions within the previous sections that using a complete video shot yields better end result than that using a key frame representing a shot while, gadget using a query clip is advanced than that the use of a single shot as an alternative. Seek based on textual information of the video can also be utilized in CBVR systems. Question with the aid of instance photograph is famous for content material based totally picture retrieval. Extending this approach for video retrieval has a challenge that motion facts of the video is not 
exploited however simplest visible information is used. Textual query becomes an option for video retrieval because it provides greater herbal interface however the end result obtained is very negative. An integrated video retrieval system in which video components are represented via more visual functions, color and movement capabilities are included to fully make the most the spatiotemporal

Records contained in a video and as a result display better consequences. Computerized retrieval systems ought to be the attention and it calls for extra interest from researchers for progressed retrieval outcomes. A fashion to reduce computational fee is wanted to mission commercialized systems for video indexing, classification and retrieval to facilitate the availability of low price, speedy and green VR systems. Functionality of these systems may be magnified through attaining large video databases that exist and are reachable on the net. The reachable databases need to empower the users with alternatives to correctly select the favored videos simplest whilst filtering out the relevant but undesired in addition to Inappropriate films so that valuable, moral, ethical and informative facts will become accessible effectively, speedy and at low value.

\section{REFERENCES}

1) Weiming Hu, Nianhua Xie, Li Li, Xianglin Zeng, Maybank S., "A Survey on Visual Content-Based Video Indexing and Retrieval", IEEE Transactions on Systems, Man, and Cybernetics, Part C (Applications and Reviews), 41-6,797-819, $11 / 2011$

2) Liang-Hua Chen, Kuo-Hao Chin, Hong-Yuan Liao, "An integrated approach to video retrieval", Proceedings of the nineteenth conference on Australasian database Volume 75, 49-55, 2008

3) Hong Jiang Zhang, Nianhua Wu, Di Zhong, Stephen W. Smoliar, "An integrated system for content-based video retrieval and browsing", Pattern Recognition, Pattern Recognition Society, Published by Elsevier Science Ltd., Vol. 30, No. 4, pp. 643 658, 1997

4) B V Patel, B B Meshram, "Content Based Video Retrieval Systems", International Journal of Unicom, vol 3, No. 2, pg 13-30, 2012

5) Yenning Deng, B.S. Manjunath, "Content-based Search of Video Using Color, Texture, and Motion", IEEE, pg 534-537, 1997
6) Ja-Hwung Su, Yu-Ting Huang, Hsin-Ho Yeh, Vincent S. Tseng , "Expert Systems with Applications", 37, pg 5068-5085, 2010

7) T.N.Shanmugham, Priya Rajendran, "An Enhanced Content-Based Video Retrieval System Based on Query Clip", International Journal of Research and Reviews in Applied Sciences, Volume 1, Issue 3, 2009

8) Ritendra Datta, Dhiraj Joshi, Jia Li, James Z. Wang, "Image Retrieval: Ideas, Influences, and Trends of the New Age", ACM Computing Surveys, Vol. 40, No. 2, article 5, pg 1-60, 2008

9) Nicu Sebe, Michael S. Lew, Arnold W.M. Smeulders, "Video retrieval and summarization", Computer Vision and Image Understanding, vol. 92 , no. 2-3, pg 141-146, 2003

10)C. V. Jawahar, Balakrishna Chennupati, Balamanohar Paluri, Nataraj Jammalamadaka, "Video Retrieval Based on Textual Queries", Proceedings of the Thirteenth International Conference on Advanced Computing and Communications, Coimbatore, Citeseer, 2005

11) Alexander G. Hauptmann, Rong Jin, and Tobun D. Ng, "Video Retrieval using Speech and Image Information", Electronic Imaging Conference (EI'03), Storage Retrieval for Multimedia Databases, Santa Clara, CA, January 20-24, 2003.

12) Steven C.H. Hoi, Michael R. Lyu, "A multimodal and multilevel ranking frame work for contentbased video retrieval", ICASSP, 2007

13) M. Petkovic, W. Jonker, "Content-Based Video Retrieval by Integrating Spatio-Temporal and Stochastic Recognition of Events", Proceedings of IEEE Workshop on Detection and Recognition of Events in Video, pp. 75-82, 2001. 